\title{
Análise das técnicas de Gamificação em Ambientes Virtuais de Aprendizagem
}

\author{
Ana Carolina Tomé Klock - actklock@gmail.com \\ Mayco Farias de Carvalho - maycofarias.joi@gmail.com \\ Brayan Eduardo Rosa - brayan_sx@hotmail.com \\ Isabela Gasparini - isabela.gasparini@udesc.br \\ Departamento de Ciência da Computação - Universidade do Estado de Santa Catarina (UDESC) \\ Joinville - SC
}

\begin{abstract}
Resumo. A gamificação está relacionada ao uso de elementos de jogos em contextos não relacionados com jogos. Atualmente, diferentes áreas fazem uso de sistemas gamificados, tais como entretenimento, saúde e especialmente a educação, uma vez que a gamificação fornece uma alternativa para engajar e motivar os estudantes durante o processo de aprendizagem. Este trabalho tem como objetivo investigar as técnicas de gamificação existentes e analisar quais técnicas são utilizadas nos Ambientes Virtuais de Aprendizagem (AVAs). Dez diferentes AVAs foram investigados. Como resultado, das onze diferentes técnicas levantadas na literatura, seis estão presentes nos ambientes estudados. Desta forma, percebe-se o potencial da gamificação relacionada à educação.
\end{abstract}

Palavras-chave: AVA, gamificação, engajamento, motivação.

\section{Analysis of gamification techniques in Virtual Learning Environments}

Abstract. Gamification is related to the use of game elements in non-game contexts. Currently, different fields use gamified systems, such as entertainment, health, and especially education, since gamification provides an alternative to engage and motivate students during the learning process. This work aims to investigate the gamification techniques and analyze which existing techniques are used in Virtual Learning Environments (VLEs). Ten VLEs were investigated. As a result, among eleven different techniques of literature, six are present in the studied environments. In this way, it is noticed the potential of the gamification related to educational field.

Keywords: VLE, gamification, engagement, motivation

\section{Introdução}

A educação sofreu diversas modificações nos últimos anos. Partiu-se de um cenário de cursos presenciais que utilizavam objetivismo com uma didática centrada no professor para cursos híbridos e on-line que utilizam tecnologias digitais para dar apoio ao construtivismo, a colaboração e a uma pedagogia centrada no estudante, além de operar em escala global (Hiltz e Turoff, 2005). Os Ambientes Virtuais de Aprendizagem (AVAs) podem ser entendidos como a utilização de multimídias tecnológicas e da Internet para melhorar a qualidade do aprendizado através da facilitação de acesso aos recursos e serviços para a troca e a colaboração (Comissão Europeia, 2001). Holmes e Gardner (2006) resumem os AVAs como o "acesso on-line aos recursos de aprendizado, em qualquer lugar e em qualquer tempo".

A utilização da tecnologia apoia a educação em diversos aspectos, porém, um antigo problema relacionado à motivação e ao engajamento dos estudantes ainda persiste nos ambientes. No sistema tradicional de ensino, a desmotivação dos alunos é resultado de diferentes aspectos, tais como a falta de compreensão do plano de ensino do curso, a didática do professor, a dificuldade em visualizar os benefícios de ir à escola, a falta de confiança, bullying, medo, cansaço, etc. (Atkin, 2012). Nos AVAs, a situação não é muito diferente. De acordo com Visser, Plomp, Amirault e Kuiper (2002), mesmo com a mediação da tecnologia para um aprendizado efetivo, os estudantes acabam enfrentando o mesmo problema motivacional do sistema tradicional de ensino, sendo inclusive uma das principais causas dos alunos desistirem do curso.

Diversas iniciativas vêm sendo estudadas para melhorar a motivação e o engajamento do estudante. Dentre elas, uma nova abordagem é a Gamificação (do 
inglês Gamification). Para Zichermann e Cunningham (2011), a gamificação é um processo de pensamento e mecânica do jogo para envolver os usuários e resolver problemas. Nesta mesma linha, Kapp (2012) define gamificação como o uso de mecanismos, estética e pensamento dos jogos para engajar as pessoas, motivar ações, promover conhecimento e resolver problemas. A Gamificação pode ser resumida como o uso de elementos de jogos em contextos não relacionados com jogos (Deterding et. al, 2011), (Cunha, 2014). A gamificação pode ser encontrada em inúmeros tipos de aplicações, desde sistemas para melhorar a produtividade na indústria, finanças, saúde, entretenimento, sustentabilidade e na educação.

A maioria dos elementos de jogos aplicados na gamificação estão diretamente relacionados aos desejos humanos, por exemplo: pontos são conectados com a necessidade de recompensa; níveis são úteis para demonstrar status; desafios permitem concluir realizações; rankings estimulam a competição; presentes deixam que as pessoas pratiquem a solidariedade (altruísmo); entre outros (BBVA Innovation Edge, 2012). A gamificação pode trazer maior engajamento e motivação para os estudantes nos AVAs, porém, o uso de gamificação na educação é ainda bastante recente. Baseando-se nisso, esse artigo tem como objetivo analisar as técnicas de gamificação e verificar quais técnicas são utilizadas nos AVAs. Para tanto, esse artigo está estruturado da seguinte forma: a seção 2 apresenta os fundamentos sobre gamificação, explorando as técnicas existentes. A seção 3 explora os AVAs gamificados. A seção 4 apresenta a análise das técnicas de gamificação encontradas nesses ambientes. Por fim, a seção 5 detalha a conclusão deste trabalho.

\section{Fundamentos sobre Gamificação}

Para entender o funcionamento da gamificação é necessário entender como um jogo funciona. Dickey (2005) afirma que os jogos possuem três partes principais: as atividades focadas em metas, o progresso do jogador e os mecanismos de recompensa (i.e. as técnicas utilizadas). Para Smith-Robbins (2011), as atividades existentes nos jogos estão tipicamente orientadas a objetivos e metas, além de uma definição clara das condições para se chegar na vitória e dos diversos obstáculos que precisam ser superados para se completar uma atividade. Observando a definição de Smith-Robbins (2011), fica bastante claro a similaridade entre jogos e o aprendizado e entre jogadores e alunos, sendo direcionados e instigados a completarem tarefas para atingirem diferentes objetivos, como vencer um jogo ou ganhar uma boa nota em alguma avaliação escolar.

Segundo Deci e Ryan (2004), a partir da teoria da autodeterminação, há três necessidades básicas da motivação intrínseca: competência, autonomia e a sensação de se pertencer a uma comunidade. Trabalhando com pontuações e níveis de experiência, o usuário é instigado a buscar as atividades a fim de cumprir metas e atingir objetivos. Deixar que os usuários criem desafios e tarefas é uma forma de autonomia que os fazem interagir com o sistema e dialogarem entre si, assim, aumentando o senso de amizade e comunidade entre os usuários/alunos. Para Glover (2013), assim como num processo de aprendizagem de um aluno, em um sistema gamificado ou em um jogo é necessário rastrear o progresso de um "jogador”, porque sem isso, seria impossível identificar as tarefas e metas que ainda faltam para o jogador chegar em uma condição de vitória (ou no caso de ambientes educacionais - aprendizagem). Uma forma de medir esse progresso é através dos mecanismos de recompensa. Assim como no processo educacional, o feedback do usuário irá definir como o mesmo está indo, e se o método da gamificação está atingindo seu objetivo central, i.e. a aprendizagem.

\subsection{Técnicas de gamificação}

A mecânica de um sistema gamificado é composta de ferramentas que, quando utilizadas corretamente, prometem um retorno significativo dos usuários (Zichermann e 
Cunningham, 2011). As principais técnicas utilizadas para a gamificação de ambientes estão descritas nas próximas subseções.

\subsubsection{Pontos}

Os sistemas de Pontos são abertos, diretos e motivacionais, permitindo a utilização de vários tipos diferentes de pontuação, de acordo com o objetivo proposto. Seus tipos são descritos a seguir, com base em Zichermann e Cunningham (2011):

- Pontos de experiência: Também conhecidos como XP, é o mais importante sistema de pontos, não serve como moeda de troca no sistema, todas as ações do jogador geram XP e uma vez adquirido ele não os perde. Apesar disso, em alguns sistemas, pontos podem expirar e/ou serem trocados. O objetivo principal é observar, classificar e guiar o usuário sobre o seu comportamento. Uma característica importante é que normalmente o sistema não possui um limite máximo de pontuação;

- Pontos resgatáveis: Também conhecidos como RP, podem ser trocados no sistema por itens que o usuário precise. Ele acumula os pontos e à medida que adquire os itens, o saldo total de pontos é atualizado. Estes tipos de pontos formam uma economia virtual, chamadas de moedas, dinheiro, energia, etc.;

- Pontos de habilidade: Também conhecidos como pontos skill, são atribuídos a tarefas específicas, que não estão relacionados às atividades principais do sistema. O usuário pode optar por realizá-las ou não, e são um conjunto bônus de pontos que permitem que o usuário ganhe experiência ou recompensas pela realização;

- Pontos de carma: São pontos que pertencem ao usuário e que ele pode compartilhar com outra pessoa. Este é o propósito deste tipo de ponto, fazendo com que o usuário seja beneficiado somente se existir compartilhamento entre as partes. Seu objetivo é criar um caminho comportamental para o altruísmo e recompensa entre usuários incentivando o compartilhamento dos pontos;

- Pontos de reputação: É o sistema mais complexo existente, é utilizado quando há a necessidade de confiança entre duas ou mais partes. Seu objetivo é indicar o quão confiável um usuário pode ser.

\subsubsection{Níveis}

Os níveis dos jogos (ou dos sistemas gamificados) indicam o progresso do usuário dentro do sistema (Zichermann e Cunningham, 2011). Existem três tipos diferentes de níveis que são descritos a seguir (Kapp, 2012):

- Níveis de jogo: Espera-se que um sistema de níveis de jogo atenda três objetivos principais. O primeiro é manter a percepção de que há progresso na história do jogo/sistema, proporcionando o engajamento do usuário. Fazendo essa relação com os ambientes educacionais, espera-se que os estudantes percebam seu progresso no processo de ensino-aprendizagem. O segundo é focado no desenvolvimento das habilidades do usuário e visa que a cada nível avançado, habilidades aprendidas sejam reforçadas ou novas sejam aprendidas, gerando aprendizagem gradativa. Por último, os níveis servirão como motivação para os usuários pois, ao avançar de nível, ele vai querer alcançar os novos objetivos do nível atual e avançar para níveis mais difíceis;

- Níveis de dificuldade: O desenvolvimento de um jogo que sempre oferece a mesma dificuldade pode criar um problema. Se um jogo/sistema for muito fácil, afastará pessoas que gostam de ser desafiados e, se for muito difícil, afastará pessoas que prefiram jogos que aumentam gradativamente a dificuldade (Kapp, 2012). Para contornar esta situação, uma prática comum dos designers é o 
desenvolvimento de três níveis de dificuldade; fácil - para iniciantes com desafios mais simples; médio - onde a maior parte dos usuários se encontra e proporciona um sistema com dificuldade e desafios equilibrados; difícil - mais indicado para usuários experientes, pois possuem desafios com maior complexidade.

- Níveis de jogador: Conhecidos como níveis de experiência, demonstram o progresso do jogador/usuário no sistema e são atribuídos ao usuário de acordo com a realização de tarefas, missões, desafios e por sua fidelização com o sistema. Geralmente esses níveis são acumulados e utilizados para alcançar habilidades especiais, adquirir itens novos ou como moeda dentro do ambiente. Os usuários valorizam atingir níveis mais altos e obter mais pontos, pois cada novo nível é mais difícil que o anterior e isso gera sensação de domínio e de realização (Kapp, 2012).

\subsubsection{Rankings}

Seu propósito principal é a comparação entre os jogadores/usuários envolvidos. Serve como uma forma de visualizar a progressão dos usuários dentro do ambiente e gera um senso de competição entre eles. Existem dois tipos de rankings:

- Ranking que não 'desincentiva': Com o advento das redes sociais, os rankings passaram a contar com uma de suas principais características que é a promoção do incentivo social. Assim, o usuário geralmente será colocado em alguma posição do ranking, conforme seu desempenho, tendo acesso às informações dos usuários mais próximos (melhores e piores que ele) ou ainda a colocação de seus amigos;

- Ranking infinito: Dependendo da quantidade de usuários de um ambiente, não há uma forma de exibi-los todos em um ranking, além disso há situações em que o usuário pode ter feito uma pontuação muito alta ou muito baixa, fazendo com que ele fique "preso" em uma posição. Desta forma, o objetivo principal desse tipo de ranking é fazer com que o usuário não fique preso em uma posição e/ou que venha a ser ultrapassado por muitos outros usuários em pouco tempo. Para alcançar este objetivo, o ranking pode ser dividido em categorias diferentes que farão com que o usuário intercale sua posição.

\subsubsection{Desafios e Missões}

Os desafios e missões são os elementos que orientam os usuários sobre as atividades que devem ser realizadas dentro de um sistema (Fadel et. al, 2014), (Zichermann e Cunningham, 2011). É importante que existam desafios para os usuários completarem, pois isso fará com que exista algo interessante para ele realizar enquanto interage com o sistema. Alguns usuários vão tentar jogar todos os desafios possíveis (sequencialmente) para finalizar todos, outros vão realizar apenas os que foram impostos para avançar no sistema. Por este motivo, o ambiente pode disponibilizar vários desafios e missões, para que o usuário permaneça motivado (Zichermann e Cunningham, 2011).

\subsubsection{Medalhas/Conquistas}

Medalhas são uma versão mais robusta de pontos e tratam-se de uma representação visual de alguma realização/conquista do usuário no sistema (Werbach e Hunter, 2012). Os usuários querem receber medalhas dentro de um ambiente por diversos motivos, para muitos, o objetivo é a experiência agradável de receber a medalha ou por “colecionar” medalhas. Do ponto de vista dos designers, as medalhas são uma forma de encorajar promoção social dos produtos e serviços (Zichermann e Cunningham, 2011). 


\subsubsection{Integração}

Integração é o ato de fazer com que uma pessoa nova ou inexperiente na aplicação seja inserida no sistema (Zichermann e Cunningham, 2011). Trata-se de um parâmetro que indica o desenvolvimento e engajamento do usuário ao estabelecer contato com o ambiente pela primeira vez. Nos primeiros minutos dentro do ambiente, a complexidade do sistema deve ser revelada aos poucos, reforçando positivamente o usuário, criando um ambiente que aceite pequenas falhas e que seja possível aprender algo sobre o indivíduo (Fadel et. al, 2014). Este item é importante para cativar e encorajar o usuário a permanecer em um sistema que até então era desconhecido e seu principal benefício é o engajamento do usuário por um longo prazo (Fadel et. al, 2014).

\subsubsection{Loops de engajamento}

Loops de engajamento estão relacionados à criação e manutenção de emoções motivadoras que contribuem para que o usuário se mantenha motivado e engajado na utilização do ambiente (Fadel et. al, 2014), não apenas no momento atual, mas também que faça com que ele tenha vontade de voltar a utilizar o jogo (Zichermann e Cunningham, 2011).

\subsubsection{Personalização}

Caracteriza-se pela possibilidade que o usuário tem de transformar e personalizar itens que compõem o sistema de acordo com o seu gosto, promovendo motivação, engajamento, sentimento de posse e controle sobre o sistema. Porém, é necessário balancear a quantidade destes itens que podem ser transformados e personalizados para que a motivação não venha a se transformar em desmotivação (Zichermann e Cunningham, 2011), (Fadel et. al, 2014).

\subsubsection{Reforço e feedback}

Reforço e feedback são recursos utilizados para prover dados importantes ao usuário, informando sua localização no ambiente e os resultados das ações realizadas por ele dentro do sistema. O retorno ao usuário é fundamental para dar suporte à sua tomada de decisão frente a situações que podem causar uma falha e para um melhor aproveitamento do usuário dentro do sistema, ou ainda para aumentar seu nível de engajamento (Fadel et. al, 2014).

\subsubsection{Regras}

Um sistema gamificado pode ser composto por um conjunto de regras que definem como o usuário pode utilizar o ambiente, como esse ambiente funciona, o que é ou não permitido, etc. As regras servem para limitar as ações dos usuários e tornar o sistema gerenciável (Kapp, 2012). As regras podem ser divididas em operacionais, que definem como o sistema funciona; formativas, que definem a estrutura formal da funcionalidade do ambiente; implícitas, que determinam o contrato/acordo entre dois ou mais usuários; e instrutivas, que determinam a forma de aprendizado (Kapp, 2012).

\subsubsection{Narrativa}

Através da narrativa pode-se fazer com que o usuário apresente um comportamento esperado frente a um contexto. Histórias são utilizadas para transmitir informações e guiar pessoas, a combinação do sistema com a história cria uma experiência interativa que engaja o usuário (Kapp, 2012).

\section{O uso de gamificação em Ambientes Virtuais de Aprendizagem}

Neste trabalho foram levantados dez diferentes AVAs que utilizam técnicas de gamificação: Khan Academy, PeerWise, QizBox, BrainScape, Peer2PeerUniversity, URI Online Judge, CodeSchool, Duolingo, Passei Direto e MeuTutor.

O Khan Academy é um ambiente educacional gratuito que disponibiliza conteúdos de Matemática, Física, Química, Biologia, Economia, Artes e Computação, 
além de possuir preparatórios para exames específicos (Khan Academy, 2014). O Khan Academy conta com vídeos hospedados no Youtube para lecionar o conteúdo, diversos exercícios para praticar o conteúdo aprendido e também permite que os pais e professores acompanhem o processo de ensino-aprendizagem do aluno. O PeerWise é um repositório gratuito e on-line de perguntas de múltipla escolha criadas, respondidas, avaliadas e discutidas pelos estudantes. Inicialmente, o próprio professor cria a página para o curso que irá lecionar e informa aos alunos para se cadastrarem no curso. A plataforma foi criada pela universidade de Auckland (Nova Zelândia) e já é utilizada para cursos como antropologia, biologia, química, computação, matemática, medicina e física (PeerWise, 2014).

O QizBox é uma aplicação web gratuita criada pela Universidade Estadual de Bowling Green (Estados Unidos) para melhorar o aprendizado nas salas de aula, permitindo que o professor apresente seus slides, realize perguntas aos alunos que estão assistindo a apresentação e forneça um feedback em tempo real (QizBox, 2014). Também existe a possibilidade de criar uma conversa on-line para os alunos discutirem sobre a lição aprendida, perguntarem e responderem questões e também realizarem anotações para quando forem revisar o conteúdo. O BrainScape é uma plataforma de estudo web e móvel para aprender mais rapidamente através da simplificação e aceleração do processo de aprendizagem. A plataforma, que possui cursos gratuitos e pagos, realiza perguntas aos alunos e permite a visualização das respostas quando os alunos acharem que estão prontos para visualizá-las, permitindo assim que os alunos tenham o aprendizado baseado na repetição, recordação ativa e meta cognição (BrainScape, 2014).

O Peer2PeerUniversity (P2PU) é um projeto de educação que aproveita a Internet e os materiais educativos disponibilizados de forma gratuita e on-line para criar um modelo de aprendizagem que auxilia o ensino superior formal tradicional (Peer2PeerUniversity, 2014). O P2PU atualmente separa os cursos oferecidos por escolas: Escola da Inovação Social, Escola de WebCraft (apoiado pela Mozilla), Escola de Conhecimento de Todos (coordenada pela CreativeCommons), Escola da Educação (focada em pedagogia) e Escola do Futuro Matemático. O URI Online Judge é um projeto gratuito realizado pela Universidade Regional Integrada (Brasil) para promover a programação de computadores na prática e auxiliar no compartilhamento do conhecimento (URI Online Judge, 2014). Esse projeto propõe problemas e permitem que os estudantes resolvam tais problemas com algoritmos nas linguagens $\mathrm{C}++$ ou Java.

O CodeSchool é uma plataforma de aprendizado on-line que ensina diversas linguagens de programação e design para web através de vídeos e exercícios interativos (CodeSchool, 2014). Apenas alguns cursos oferecidos pela plataforma são gratuitos. O Duolingo é a maior plataforma on-line e gratuita em educação de idiomas, com 38 milhões de usuários e 17 idiomas diferentes disponíveis. Essa plataforma foi criada pela Universidade de Carnegie Mellow (Estados Unidos) (Duolingo, 2014). O Passei Direto é uma rede social colaborativa gratuita para conectar estudantes e compartilhar conhecimento, contando com mais de 2 milhões de usuários (Passei Direto, 2014). Criada pela Pontifícia Universidade Católica do Rio de Janeiro em 2012, a rede social permite que os alunos organizem sua grade de disciplinas (ensino médio e superior), conheçam outros estudantes, tirem dúvidas, encontrem materiais de estudo e recebam convites para empregos em grandes empresas brasileiras. O MeuTutor é uma plataforma educacional personalizada focada na qualidade do ensino e no desempenho dos alunos, criado com base em teses de doutorado nas áreas de aprendizagem colaborativa e personalizada. Segundo o MeuTutor (2014), o objetivo social da plataforma é reduzir a discrepância entre o ensino público e privado no país através do 
seu uso como ferramenta auxiliar para estudar para o Exame Nacional do Ensino Médio (ENEM). Apesar disso, no preparatório para o ENEM, apenas o ensino de matemática é gratuito. Além do preparatório, a plataforma oferece cursos técnicos e corporativos.

\section{Análise das técnicas de gamificação encontradas}

Cada ambiente educacional descrito anteriormente foi analisado em busca dos elementos de jogos que foram descritos na seção 2. Para isso, a metodologia adotada foi primeiramente o cadastro nos ambientes, passando pela inspeção por todas as áreas do ambiente, sendo estas gerais ou de um curso cadastrado, e também a observação da visão de aluno e professor, quando essa disponível no ambiente. Além disso, as tarefas existentes também foram inspecionadas. Os ambientes foram avaliados e dessa forma foram detectados as técnicas e elementos de jogos aplicados em cada ambiente. A seguir são descritas as técnicas encontradas em cada ambiente.

No Khan Academy, foi possível encontrar cinco técnicas de gamificação diferentes: a utilização de pontos, de níveis e missões e a implementação de medalhas e personalização. Os pontos de experiência são conquistados quando o aluno termina alguma atividade. Esses pontos servem para habilitar a missão para evoluir o nível de aprendizado e também para liberar os personagens para personalização do avatar do aluno. Além disso, existem medalhas que são conquistadas por pontos ou missões específicas. No PeerWise, existem quatro técnicas de gamificação: pontos, rankings, medalhas e missões. Similar ao Khan Academy, o aluno conquista medalhas através de missões completadas, mas no PeerWise também existe um ranking indicando quantos alunos já conquistaram aquela medalha anteriormente. Além disso, o PeerWise tem um ranking conforme as pontuações de reputação (que pontua perguntas criadas, respostas realizadas e a classificação atribuída às perguntas respondidas) e as respostas corretas.

No QizBox, existem duas técnicas: pontos e níveis, onde os pontos de experiência conquistados durante as tarefas e são utilizados para melhorar os níveis do aluno. No BrainScape, também foram encontradas apenas duas técnicas: pontos e rankings, onde os pontos são conquistados durante o aprendizado para calcular o progresso de quanto falta para concluir o curso e, baseando-se nesse progresso, é montado o ranking dos alunos mais avançados no conteúdo.

No P2PU existem duas técnicas: personalização, onde o usuário pode colocar sua foto de avatar, e medalhas, conquistadas durante a execução dos cursos oferecidos. No URI Online Judge existem quatro técnicas de gamificação: os rankings, as medalhas, as missões e a personalização. Tal como o P2PU, o URI Online Judge também permite que o aluno customize seu avatar. As missões permitem que o aluno conquiste diversas medalhas. Possui o ranking geral e o ranking por universidade, classificando os alunos de acordo com a quantidade de problemas resolvidos.

No CodeSchool foram encontradas as mesmas técnicas aplicadas no Khan Academy, oferecendo missões que, ao completadas, dão pontos aos alunos. Esses pontos podem ser utilizados para "comprar" respostas quando o aluno possui dificuldade em concluir uma missão. Similar ao Khan Academy, o aluno sobe de nível ao completar as missões. No CodeSchool, o aluno ganha medalhas ao completar níveis, diferentemente do Khan Academy que dá medalhas para missões e pontos. O CodeSchool também permite a customização do avatar, tal como o P2PU e o URI Online Judge. No Duolingo, existem seis técnicas de gamificação disponíveis: pontos, níveis, rankings, missões, medalhas e personalização. Os pontos conquistados podem ser de experiência ou resgatáveis (chamados lingots). Os pontos de experiência são usados para alcançar a meta diária (missão) estipulada pelo próprio aluno, enquanto os lingots podem ser usados para comprar bens virtuais (como vidas). Existem diversos níveis conforme o idioma que se está aprendendo. Alguns níveis são sequenciais e 
outros podem ser executados em paralelo, sendo que cada nível completado dá direito a uma medalha, como ocorre no CodeSchool. Além disso, existe um ranking por período baseado nos pontos de experiência adquiridos pelo aluno, sendo que esse ranking considera apenas outros estudantes que são "seguidos” pelo aluno. Como em outros AVAs citados anteriormente, o Duolingo também permite a personalização do avatar.

No Passei Direto, foi possível encontrar seis técnicas. O aluno deve completar missões para conquistar pontos de experiência e aumentar seu nível no jogo. Diferente dos demais AVAs, no Passei Direto, ao incrementar o nível, ganha-se uma medalha diferente que é exibida ao lado do avatar, sendo esse último personalizado pelo aluno. Também existe um ranking do aluno e da universidade com base nos pontos adquiridos. No MeuTutor, assim como no Duolingo e no Passei Direto, foram encontradas seis técnicas de gamificação. Também existe o conceito de missões para conquistar pontos de experiência. Esses pontos são utilizados para o incremento de nível e para a montagem do ranking. Existem missões para conquistar medalhas e permite que o aluno customize seu avatar.

A Figura 1 apresenta uma tela de cada ambiente pesquisado onde se encontram elementos de gamificação. Destacamos com retângulos coloridos algumas técnicas de gamificação utilizadas nos ambientes. As cores dos retângulos indicam qual técnica está sendo utilizada, e está relacionada a Figura 2 - azul escuro indica pontos, vermelho indica níveis, verde indica missões, roxo indica medalhas, amarelo indica ranking, e azul claro indica personalização.

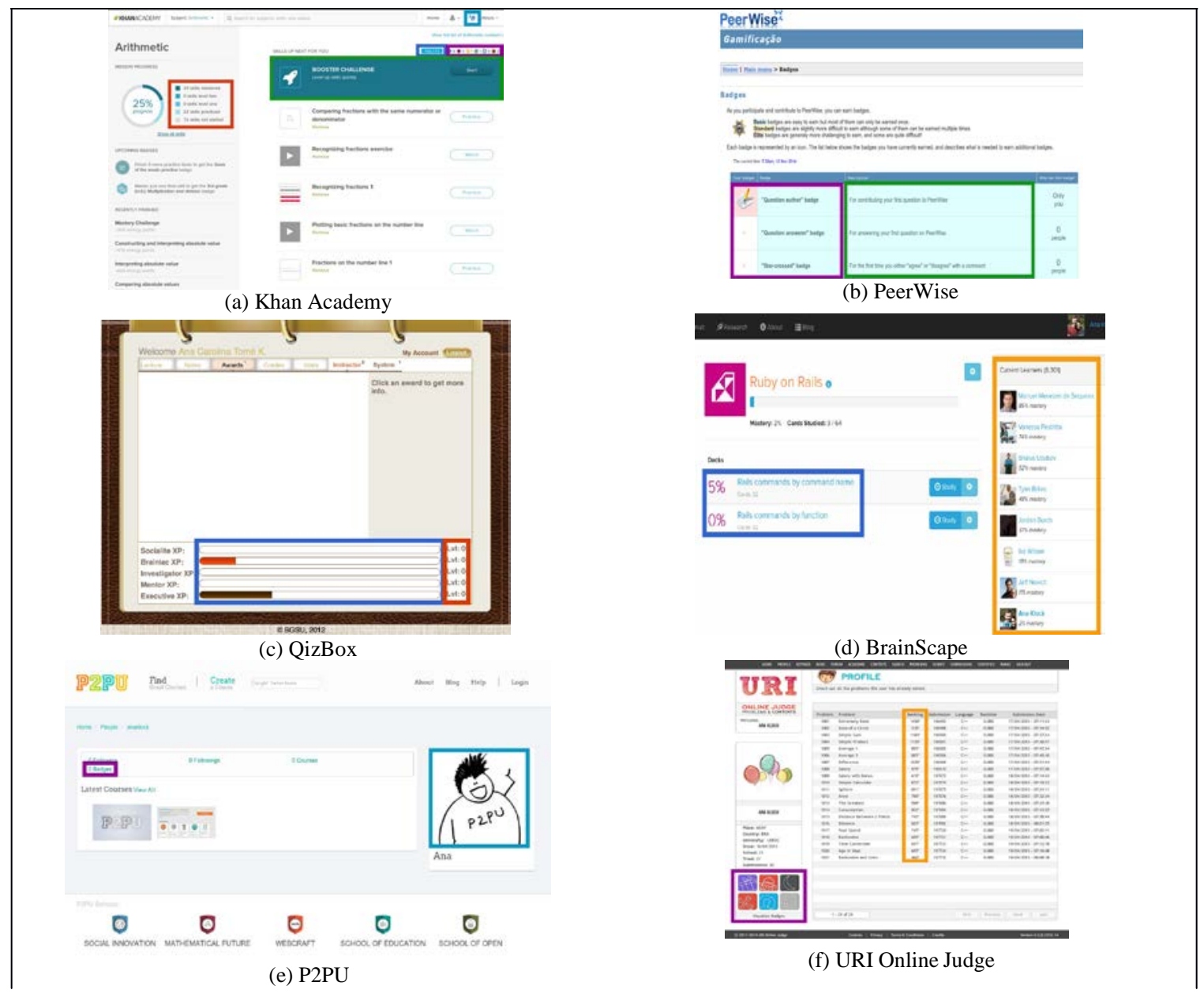




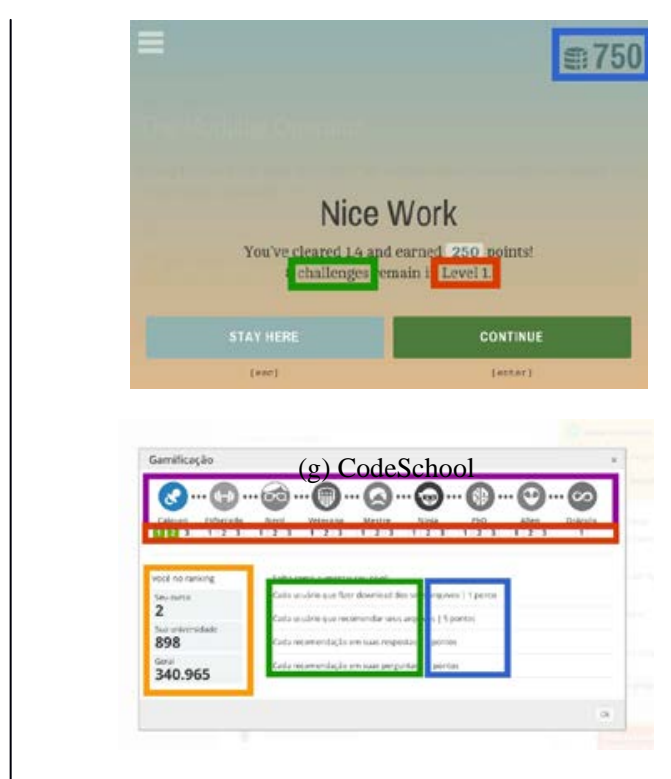

(i) PasseiDireto

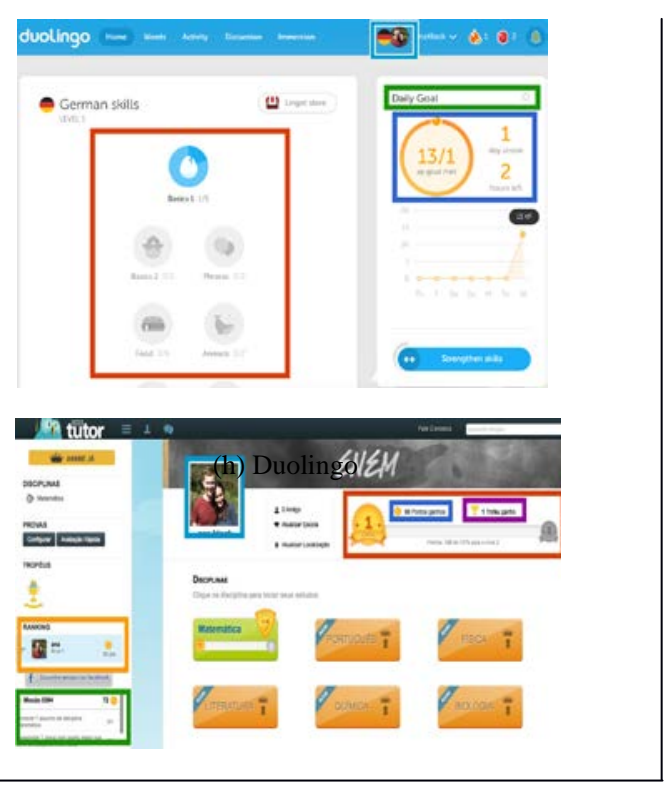

(j) MeuTutor

Figura 1. Ambientes Virtuais de Aprendizagem Gamificados

A partir dos dados e ambientes estudados, foram gerados dois gráficos que permitem uma visão geral da análise realizada. Pela Figura 2 é possível mensurar quantas técnicas de gamificação foram aplicadas em cada ambiente. Os ambientes Duolingo, Passei Direto e MeuTutor foram os ambientes que aplicaram o maior conjunto de técnicas de gamificação, seguidos pelos ambientes Khan Academy e CodeSchool.

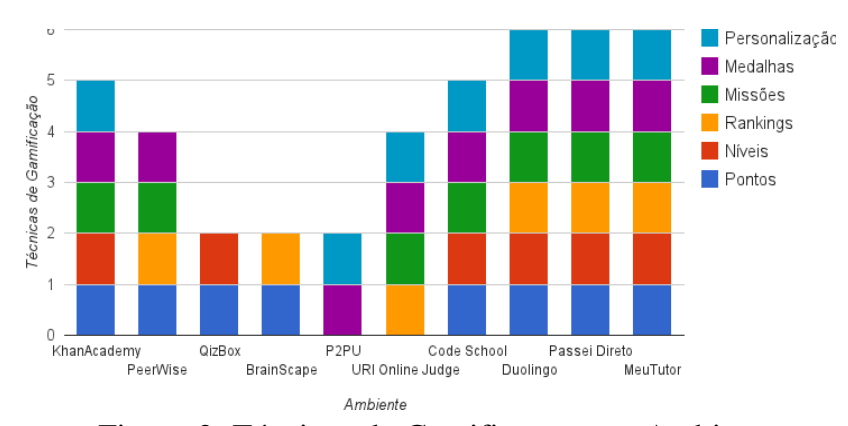

Figura 2. Técnicas de Gamificação por Ambiente

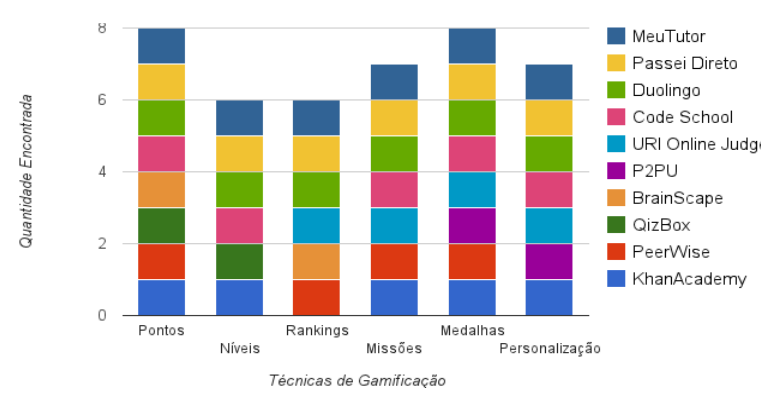

Figura 3. Técnicas de Gamificação mais aplicadas

A Figura 3 identifica quais técnicas de gamificação foram mais utilizadas ao considerar os dez ambientes analisados. Através da Figura 3 é possível visualizar que as técnicas mais utilizadas foram: Pontos e Medalhas; Missões e Personalização; e Níveis e Rankings.

\section{Conclusão}

Este trabalho teve como objetivo analisar as técnicas de gamificação existentes e verificar quais técnicas são utilizadas na educação, especificamente nos AVAs. Das onze técnicas levantadas da literatura, apenas seis estão presentes nos ambientes avaliados. Percebe-se que apesar do tema ser novo, diferentes ambientes já incorporaram técnicas de gamificação para melhorar o engajamento e a motivação dos estudantes. Esse é um problema antigo do processo de ensino-aprendizagem, e diferentes estratégias vem sendo utilizadas para aumentar a motivação dos alunos.

Como trabalho futuro, propomos um modelo conceitual para auxiliar pesquisadores e professores a acrescentarem elementos de gamificação em seus ambientes e cursos on-line com base no perfil do estudante. Este modelo visa identificar 
os principais perfis versus as técnicas de gamificação que podem auxiliar positivamente ao engajamento do aluno frente ao sistema.

\section{Referências}

ATKIN, C. Education and Minorities. London: Continuum International Publishing Group, 2012. BBVA INNOVATION EDGE. Gamification: The business of fun. Madrid: BBVA Innovation Center, 2012.

BRAINSCAPE. Site Oficial. Disponível em: <https://www.brainscape.com>. Acesso em: 08 nov. 2014. CODESCHOOL. Site Oficial. Disponível em: <https://www.codeschool.com>. Acesso em: 08 nov. 2014.

COMISSÃO EUROPÉIA. Communication from the Commission New Skills for New Jobs. 2008. CUNHA, LUCAS FELIPE DA. Modelo conceitual para a gamificação em ambientes e-learning e sua utilização no AdaptWeb ${ }^{\circledR}$. Trabalho de conclusão de curso (Bacharelado em Ciência da Computação) Departamento de Ciência da Computação, Universidade do Estado de Santa Catarina, Joinville, 2014.

DECI, Edward L.; RYAN, Richard M. Handbook of self-determination research. Rochester: University of Rochester Press, 2004.

DETERDING, S.; DIXON, D.; KHALED, R.; NACKE, L. From game design elements to gamefulness: defining gamification. In: PROCEEDINGS OF THE 15th INTERNATIONAL ACADEMIC MINDTRECK CONFERENCE, 2011, p. 9-15, Tampere.

DICKEY, Michele D. Engaging by design: how engagement strategies in popular computer and video games can inform instructional design. In: JOURNAL OFEDUCATION TRAINING RESEACH AND DEVELOPMENT, 2005.

DUOLINGO. Site Oficial. Disponível em: <https://www.duolingo.com>. Acesso em: 08 nov. 2014.

FADEL, L. M.; ULBRICHT, V. R.; BATISTA, C. R.; VANZIN T. Gamificação na educação. São Paulo: Pimenta Cultural, 2014.

GLOVER, Ian. Play as you learn: gamification as a technique for motivating learners. In: Proceedings of World Conference on Educational Multimedia, 2013, Victoria.

HILTZ, S.; TUROFF, M. Education goes digital: the revolution of online learning and the revolution in higher education. In: Communications of ACM.2005, New York.

HOLMES, B.; GARDNER, J. E-learning: Concepts and Practice. London: SAGE Publications, 2006.

KAPP, K. M. The gamification of learning and instruction: game-based methods and strategies for training and education. San Francisco: Pfeiffer, 2012.

KHAN ACADEMY. Site Oficial. Disponível em: <http://www.khanacademy.org>. Acesso em: 08 nov. 2014.

MEUTUTOR. Site Oficial. Disponível em: <http://www.meututor.com.br>. Acesso em: 08 nov. 2014.

PASSEI DIRETO. Site Oficial. Disponível em: <https://www.passeidireto.com>. Acesso em: 08 nov. 2014.

PEER2PEER UNIVERSITY. Site Oficial. Disponível em: <https://p2pu.org/en>. Acesso em: 08 nov. 2014.

PEERWISE. Site Oficial. Disponível em: <https://peerwise.cs.auckland.ac.nz>. Acesso em: 08 nov. 2014.

QIZBOX. Site Oficial. Disponível em: <http://qizbox.bgsu.edu/public/login>. Acesso em: 08 nov. 2014. SMITH-ROBBINS, S. This Game Sucks: How to Improve the Gamification of Education. Educause Review Online, 2011.

URI ONLINE JUDGE. Site Oficial. Disponível em: <https://www.urionlinejudge.com.br>. Acesso em: 08 nov. 2014.

WERBACH, K.; HUNTER, D. For the win: How game thinking can revolutionize your business. Philadelphia: Wharton Digital Press, 2012.

VISSER, L.; PLOMP, T.; AMIRAULT, R.; KUIPER, W. Motivating students at a distance: The case of an international audience. In: JOURNAL OF EDUCATIONAL TECHNOLOGY RESEARCH AND DEVELOPMENT, 2002.

ZICHERMANN, G.; CUNNINGHAM, C. Gamification by Design: Implementing game mechanics in web and mobile apps. Sebastopol: O'Reilly Media Inc, 2011. 